actually harvested? Shame on me for jumping on the learning experience!"

I didn't tell him, but he was sharing. Finally, after years of amassing pain, he was sharing; paving the way to his own recovery.

Medicine provides a privileged glimpse into the most tragic hours of humanity. Quietly, stealthily, we accumulate hundreds of lives' worth of sorrow into our own; seeing, hearing, smelling and feeling death like no other. Indeed, no one can understand or experience death like we do.

Perhaps, it is the pound of flesh we owe for the privilege of sharing a father's joy as he cuts his newborn's umbilical cord.

Perhaps it is the price of the tearful gratitude of the mother whose child you resuscitated.

Perhaps it is what we must bear in exchange for the satisfaction of having relieved an old man's pain.
Vicariously, we suffer. But perhaps, just perhaps, it is what distinguishes the healers, from the doctors.

\section{Steven Bellemare MD}

Child abuse pediatrician

Ottawa, Ont.

The author confirms that all patients in this work are fictitious.

CMAJ 2011. DOI:10.1503/cmaj.110204

\title{
No unifying theories but lots of chat
}

\author{
The Ethics of Consent: Theory and Practice \\ Franklin G. Miller, Alan Wertheimer, \\ Editors \\ Oxford University Press; 2009.
}

$\mathrm{A}$ reviewer should consider a book for what it is, rather than for what he might have wished it to be. And so a clinician reviewing a text on a subject of practical importance, such as consent, must take care not to expect a helpful manual for extricating oneself from thorny clinical brambles.

With that in mind, The Ethics of Consent, edited by National Institutes of Health bioethicists Franklin Miller and Alan Wertheimer, should be read as a philosophy book, with a particular philosophical goal. Having noted that issues of consent arise around sex in the bedroom, government in the state, and everywhere in between, the editors also observe a "regrettable lack of cross-fertilization among the different contexts." Consequently, they hope their book will "stimulate ... hybrid vigour" in the discussion of consent in its various guises.

Unfortunately, The Ethics of Consent fails to achieve its own purpose.

None of the contributing authors go so far as to claim to have developed a unified theory of consent. But stating that "... a theory of informed consent should be rooted in a theory that is adequate for the full range of consent contexts" suggests that one at least believes that such a theory is possible. However, there is little progress toward such a theory here.

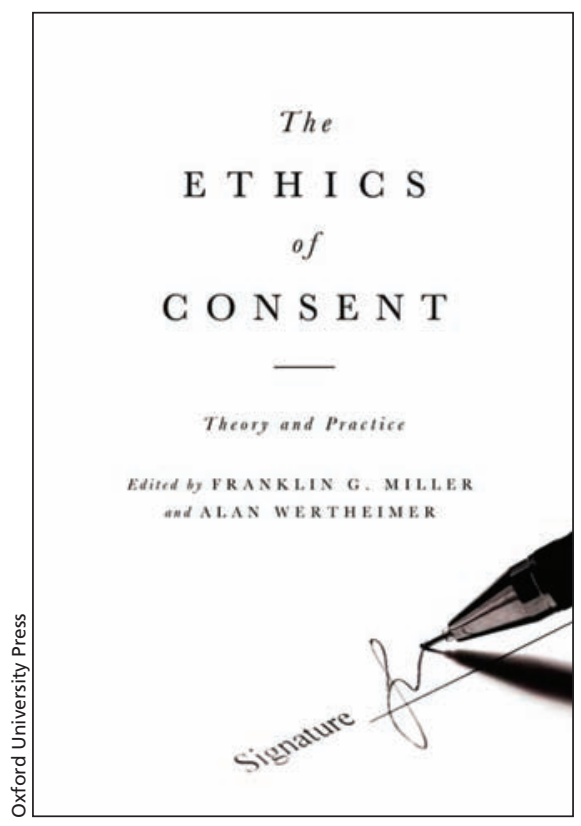

A comprehensive array of topics is covered, but presented as clearly separated subjects. This seems a peculiar decision by the editors, who lament the existing literature's lack of inter-contextual cross-talk but choose to organize their book in a way that does not engender a solution to this problem. In addition, many individual subjects are treated quite narrowly. A discussion of the legal aspects of consent to sex, for example, is largely a rebuttal of specific "liberal," "feminist," and "queer" theories. Likewise, in considering consent to medical treatment, physicians' fiduciary roles are discussed at length, but other issues, such as consent by proxies and surrogates, are mentioned only incidentally. As a sampler, rather than a survey, this book seems unlikely to create vigorous hybrid philosophies.

But it is possible that even a broader treatment would be doomed to fail. Although the term "consent" is used in a variety of contexts, the specific meaning varies widely. Is the consent that is given to a business contract or a sexual liaison substantially similar to the consent that is given to a medical treatment? The word is the same, but nearly everything else differs in important respects. Is it reasonable to expect these very different situations to have anything informative to say about one another? The ease with which the authors are able to use a standard philosopher's technique - providing counter-examples to an existing theoretic framework's reach in order to justify the construction of a new framework - suggests that an all-encompassing theory does not exist. How could any theory survive such an assault of expert and facile contradiction, especially when the underlying subject matter seems not to have any particular unity?

The Ethics of Consent does contain some interesting individual discussions, and can be usefully read as a collection of loosely connected essays. But it is not the book it wishes to be.

\section{Paul Moorehead MD}

PhD student

Pathology and Molecular Medicine

Queen's University

Kingston, Ont.

CMAJ 2011. DOI:10.1503/cmaj.110420 